\title{
INTERFERENCE SUPPRESSING RECEIVER TECHNIQUE FOR WIRELESS AD HOC NETWORKS
}

\author{
Sunho Park and Byonghyo Shim \\ Institute of New Media and Communication, Department of Electrical and \\ Computer Engineering, Seoul National University, Seoul, Korea \\ sunhopark@islab.snu.ac.kr, bshimesnu.ac.kr
}

\begin{abstract}
Recent works on ad hoc network study have shown that achievable throughput can be made to scale linearly with the number of receive antennas even if the transmitter has a single antenna. In this paper, we propose a method pursuing robustness in ad hoc network system when the channel state information (CSI) of interferers is unavailable. The non-parametric linear minimum mean square error (MMSE) filter is exploited to achieve large fraction of the MMSE filter transmission capacity employing the perfect covariance matrix information. The key feature ingredient to make our approach effective is to exploit the autocorrelation of received signal, which obtains the covariance matrix information without transmission rate loss. From the numerical results, we show that the proposed scheme brings substantial transmission capacity gain over conventional MMSE filter using sample covariance matrix.
\end{abstract}

\section{KEYWORDS}

Ad hoc network, channel estimation, receiver technique, minimum mean square error

\section{INTRODUCTION}

In the decentralized wireless network (ad hoc network), multiple transmitter-receiver pairs transmit simultaneously without the benefit of fixed infrastructure. Due to the uncoordinated nature of communication, multiple transmitters communicate simultaneously, and hence incur substantial interference which severely decreases the transmission rate. In an ad hoc wireless network, exploiting multiple receive antennas has been one of the promising solutions to increase data rate and deal with inter-user interference. Prior works on investigating the transmission capacity with multiple receive antennas [1]-[3] considered some specific multiple antenna configurations. In [1] the maximal ratio combining (MRC) only provides array gain while [2] considers full zero forcing to remove the strongest interferers but no array gain is provided. It is shown in [3] that both conventional MMSE filter and partial zero forcing (PZF) provide a benefit that network-wide throughput scales linearly with the number of receive antennas even if each transmitter has only a single antenna. All these promising gains are achieved assuming perfect channel state information at the receiver (CSIR). Although perfect CSIR is reasonable for initial state of research, further investigation of imperfect CSIR would be interesting since practical network in general has imperfect channel state information due to estimation errors. Recently, as a way to deal with imperfect CSIR issue, Jindal et al. proposed the filter employing sample covariance matrix which can be estimated by listening to the interference and noise observations [3]. However, the shortcoming of this filter using sampled covariance matrix is that data rate loss

Natarajan Meghanathan et al. (Eds) : NETCOM, NCS, WiMoNe, GRAPH-HOC, SPM, CSEIT - 2016 pp. 91-102, 2016. (C) CS \& IT-CSCP 2016

DOI : $10.5121 /$ csit.2016.61509 
is substantial since the covariance matrix is sampled in an inactive mode of the desired transmitter.

In this paper, we propose a technique that employs nonparametric linear minimum mean square error (MMSE) receive filter for improving network-wide throughput. To be specific, when the CSIR of all the interferers is unavailable, which is true for real ad hoc network scenarios, the proposed scheme exploits the autocorrelation of the received signal for MMSE operation. Even with the covariance matrix variation, the maximized SINR of the proposed method is identical to that of the conventional filter. Due to the fact that the autocorrelation of the received signal is obtained the data transmission period, the proposed method achieves large fraction of optimal transmission capacity.

The rest of this paper is organized as follows. In Section 2, we describe the system model and the summary of the conventional MMSE filter. In Section 3, we present the proposed non-parameter linear MMSE method. Simulation results and conclusion are provided in Section 4 and Section 5.

\section{AD HOC NETWORK}

\subsection{System Model}

In an ad hoc network, the active transmitters are placed according to a 2-D homogeneous Poisson point process (PPP) of density $\lambda$ ( transmitters $/ \mathrm{m}^{2}$ ). Each single transmit antenna communicates with a receiver equipped with $N$ antennas, where each receiver is randomly located at $d$ meters away from the corresponding transmitter. Due to the Poisson process stationarity, we focus on a typical transmit-receive pair denoted by $T x_{d}$ and $R x_{d}$, respectively. From the viewpoint of $R x_{d}$, the set of interferers except $T x_{d}$ also sets a homogeneous PPP due to Slivnyak's Theorem [4]. The set of all the active transmitters is denoted by denoted by $\mathrm{A}=\left\{\left(X_{i}, \mathbf{h}_{i}\right),\left(d, \mathbf{h}_{d}\right), \lambda, i \in \mathbf{N}\right\}$ where $X_{i}$ and $\mathbf{h}_{i}$ are the location and channel vector of the $i$ th transmitting node with respect to the typical receiver.

Under the frequency-flat channel, the received signal $\mathbf{y}$ is

$$
\mathbf{y}=d^{-\alpha / 2} \mathbf{h}_{d} s_{d}+\sum_{i \in \mathrm{A}(\lambda) \backslash\left\{X_{d}\right\}}\left|X_{i}\right|^{-\alpha / 2} \mathbf{h}_{i} s_{i}+\mathbf{w}
$$

where $\alpha(>2)$ is a path-loss exponent, $\left|X_{i}\right|$ is the distance from the desired transmitter to the $i$ th interferer, $\mathbf{h}_{i}$ is the channel vector $(N \times 1)$ from the $i$ th interferer to the desired receiver, $\mathbf{w}$ is the complex Gaussian noise vector $\left(\mathbf{w} \sim C N\left(0, \sigma^{2} \mathbf{I}\right)\right)$, and $s_{i}$ is the symbol transmitted by the $i$ th interferer $\left(E\left[\left|s_{i}\right|^{2}\right]=\rho\right)$. Without loss of generality, we assume that the distances $\left|X_{i}\right|$ are ordered so that the squared-distances $\left|X_{1}\right|^{2},\left|X_{2}\right|^{2}, \ldots$ follow the 1-D PPP with intensity $\pi \lambda$ [5]. 


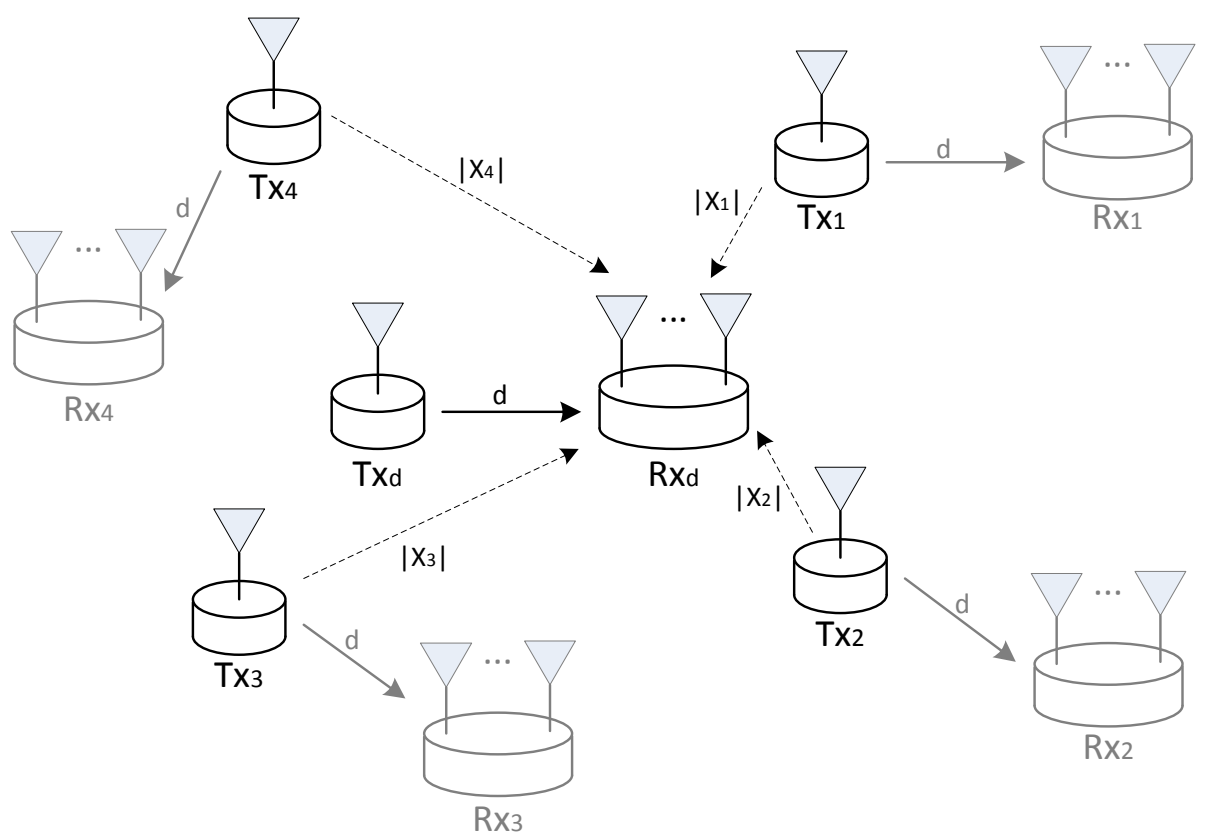

Figure 1. Desired Transmit-receive pair with interferers

\subsection{Conventional MMSE Receiver}

In this subsection, we describe the transmission capacity for the ad hoc network and review the conventional MMSE filter. With the inclusion of unit norm receive filter $\mathbf{v}_{d}$, the estimated symbol becomes $\hat{s}_{d}=\mathbf{v}_{d}^{H} \mathbf{y}$ and hence resulting signal-to-interference-and-noise ratio (SINR) is

$$
\operatorname{SINR}=\frac{\rho d^{-\alpha} \mathbf{v}_{d}^{H} \mathbf{h}_{d} \mathbf{h}_{d}^{H} \mathbf{v}_{d}}{\mathbf{v}_{d}^{H}\left(\sigma^{2} \mathbf{I}+\rho \sum_{i \in \mathrm{A}(\lambda)}\left|X_{i}\right|^{-\alpha} \mathbf{h}_{i} \mathbf{h}_{i}^{H}\right) \mathbf{v}_{d}} .
$$

When all transmitters send at the rate equal to $R=\log _{2}(1+\beta)$, a communication is regarded as successful if and only if the received SINR is larger than $\beta$. Hence the outage probability at SINR threshold $\beta$ is $P_{\text {out }}(\lambda)=P[\operatorname{SINR} \leq \beta]$, which is an increasing function of $\lambda$. By the stationarity of the process, this outage probability approximates the network-wide packet error probability. Further, the maximum interferer density such that the outage does not exceed $\varepsilon(>0)$

is $\lambda_{\varepsilon}=\max _{\lambda}\left\{\lambda: P_{\text {out }}(\lambda) \leq \varepsilon\right\}$ where $\varepsilon$ is a constant outage level, ensuring a typical transmission will succeed with probability $1-\varepsilon$. Then, the transmission capacity of the ad hoc network is

$$
C(\varepsilon)=\lambda_{\varepsilon}(1-\varepsilon) \log _{2}(1+\beta) \quad \mathrm{bps} / \mathrm{Hz} / m^{2},
$$

by accumulating all the $\lambda_{\varepsilon}$ simultaneous transmissions in the network [1], [6], [7].

Due to the fact that the SINR and $\lambda_{\varepsilon}$ primarily depend on the receive filter, multiple receive antenna technique has received much attention as a means to mitigate interference [3], [7], [8]. It is well known that the MMSE filter optimally pursues balance between signal boost and 
interference suppression for maximizing the SINR [3]. The normalized MMSE receive filter is given by

$$
V_{d}=\frac{\Sigma^{-1} \mathbf{h}_{d}}{\left\|\Sigma^{-1} \mathbf{h}_{d}\right\|}
$$

where $\Sigma=\frac{1}{S N R} \mathbf{I}+d^{\alpha} \sum_{i \in \mathrm{A}(\lambda) \backslash T x_{d}}\left|X_{i}\right|^{-\alpha} \mathbf{h}_{i} \mathbf{h}_{i}^{H}$ is the spatial covariance of the interference plus noise and $S N R=\frac{\rho d^{-\alpha}}{\sigma^{2}}$. Using (4) and (2), the maximized received SINR of the MMSE filter becomes

$$
\begin{aligned}
\operatorname{SINR}_{M M S E} & =\frac{\rho d^{-\alpha}\left(\mathbf{h}_{d}^{H} \Sigma^{-1} \mathbf{h}_{d}\right)^{2}}{\mathbf{h}_{d}^{H} \Sigma^{-1}\left(\sigma^{2} \mathbf{I}+\rho \sum_{i \in \mathrm{A}(\lambda)}\left|X_{i}\right|^{-\alpha} \mathbf{h}_{i} \mathbf{h}_{i}^{H}\right) \Sigma^{-1} \mathbf{h}_{d}} \\
& =\mathbf{h}_{d}^{H} \Sigma^{-1} \mathbf{h}_{d} .
\end{aligned}
$$

When the CSIR of all the interferers is unavailable, the receiver should estimate the interfering channels information to design the optimal MMSE filter. Note that since the desired channel can be estimated accurately via pilot symbols, the primary concern of the ad hoc network is the interfering channels estimation. The MMSE with imperfect CSIR [3] estimates the sampled covariance matrix by listening to interferer transmissions in the absence of desired signal. If the desired transmitter remains inactive for $K$ symbols duration, the receiver can employ the $K$ observations to organize the sample covariance as

$$
\hat{\Sigma}=\frac{1}{K} \sum_{i=1}^{K} \mathbf{r}_{i} \mathbf{r}_{i}^{H}
$$

where $\mathbf{r}_{i}$ represents the $i$ th observation including interference and noise. By replacing $\hat{\Sigma}$ with $\Sigma$ in (4), the resulting SINR becomes SINR $=\frac{\left(\mathbf{h}_{d}^{H} \hat{\Sigma}^{-1} \mathbf{h}_{d}\right)^{2}}{\mathbf{h}_{d}^{H} \hat{\Sigma}^{-1} \Sigma \hat{\Sigma}^{-1} \mathbf{h}_{d}}$. Under the assumption that all the interferers send independent Gaussian symbols, the expected SINR with respect to the $\hat{\Sigma}$ distribution is [9]

$$
E\left[\frac{\left(\mathbf{h}_{d}^{H} \hat{\Sigma}^{-1} \mathbf{h}_{d}\right)^{2}}{\mathbf{h}_{d}^{H} \hat{\Sigma}^{-1} \Sigma \hat{\Sigma}^{-1} \mathbf{h}_{d}}\right]=\left(1-\frac{N-1}{K+1}\right) \mathbf{h}_{d}^{H} \Sigma^{-1} \mathbf{h}_{d} .
$$

\section{NON-PARAMETRIC LINEAR MMSE RECEIVER}

In this section, we present the proposed filter based on the non-parametric linear MMSE estimation. There are three following drawbacks in the conventional MMSE receiver. First, when the receiver estimates $K$ observations, the desired transmitter should be turned off for $K$ symbols duration. The training duration will be substantial and cause the substantial transmission data rate loss even though the receiver provide good covariance matrix for sufficient $K$. Second, the covariance matrix information should be updated when the channel state is changed. If the 
channel is changing per $T$ symbols period, the effective rate is decreased by the factor of $\frac{T-K}{T}$. Finally, $K$ should increase to attain the identical SINR in (5) when the $N$ increases (see Fig. 2).

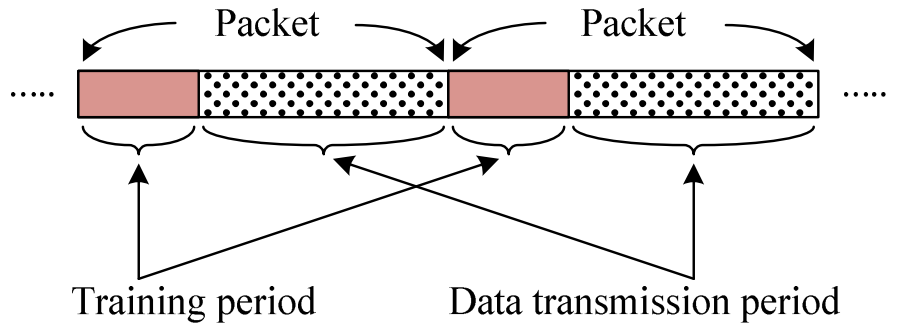

(a)

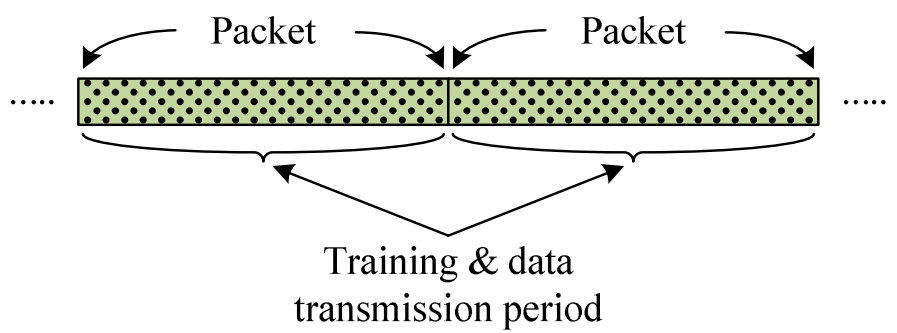

(b)

Figure 2. Packet structure: (a) conventional method and (b) proposed method.

\subsection{Non-Parametric Linear MMSE}

The key distinction of the proposed method over the conventional MMSE filter is that the desired channel information is additionally incorporated on top of the observations of the interference and noise. From [10], the estimated desired symbol of the linear MMSE is given by

$$
\begin{aligned}
\hat{s}_{d} & =\mathbf{v}_{d}^{H} \mathbf{y}=R_{s_{d} \mathbf{y}} R_{\mathrm{yy}}^{-1} \mathbf{y} \\
& =\mathbf{h}_{d}^{H}\left(\frac{1}{S N R} \mathbf{I}+\sum_{i \in \mathrm{A}(\lambda) \backslash\left\{x_{d}\right\}} \mathbf{h}_{i} \mathbf{h}_{i}^{H}+\mathbf{h}_{d} \mathbf{h}_{d}^{H}\right)^{-1} \mathbf{y} \\
& =\mathbf{h}_{d}^{H}\left(\Sigma+\mathbf{h}_{d} \mathbf{h}_{d}^{H}\right)^{-1} \mathbf{y} .
\end{aligned}
$$

Following theorem explains the fact that the linear MMSE filter in (8) can achieve the maximum SINR of (5) regardless of the inclusion of the desired channel information.

Theorem 3.1: The linear MMSE filter using non-parametric autocorrelation of the received signal $R_{\mathrm{yy}}$ is

$$
\mathbf{v}_{d}=\left(\Sigma+\mathbf{h}_{d} \mathbf{h}_{d}^{H}\right)^{-1} \mathbf{h}_{d}
$$

and the corresponding SINR becomes 


$$
\operatorname{SINR}=\mathbf{h}_{d}^{H} \Sigma^{-1} \mathbf{h}_{d}
$$

Employing the Sherman-Morrison formula [10], the linear MMSE receiver with the autocorrelation of the received signal achieves the maximum transmission capacity. By modifying the Theorem 3.1, the SINR of the proposed algorithm under imperfect CSIR condition is obtained. The sample covariance of the proposed receiver is $\hat{\Sigma}_{d}=\hat{\Sigma}+\mathbf{h}_{d} \mathbf{h}_{d}^{H}$ where $\hat{\Sigma}$ represents the observations of the noise plus interference. With the knowledge of $\mathbf{h}_{d}$, the receiver can compute the filter $\hat{\mathbf{v}}_{d}=\hat{\Sigma}_{d}^{-1} \mathbf{h}_{d}$ and the resulting SINR becomes

Following theorem explains the fact that the linear MMSE filter in (8) can achieve the maximum SINR becomes

$$
\operatorname{SINR}_{\text {prop }}=\frac{\left(\mathbf{h}_{d}^{H} \hat{\Sigma}_{d}^{-1} \mathbf{h}_{d}\right)^{2}}{\mathbf{h}_{d}^{H} \hat{\Sigma}_{d}^{-1} \Sigma \hat{\Sigma}_{d}^{-1} \mathbf{h}_{d}} .
$$

One can show that the expected SINR is

$$
E\left[\operatorname{SINR}_{\text {prop }}\right]=\left(1-\frac{N-1}{M+1}\right) \mathbf{h}_{d}^{H} \Sigma^{-1} \mathbf{h}_{d} .
$$

Note that the (12) based upon the sample covariance $\hat{\Sigma}_{\text {prop }}$ is precisely a factor of $1-\frac{N-1}{M+1}$ smaller than the expected SINR with perfect knowledge of $R_{\mathrm{yy}}$. This factor is increasing in $M$ and converges to one as $M \rightarrow \infty$ because $\hat{\Sigma}_{\text {prop }} \rightarrow \Sigma_{\text {prop }}$ as $M \rightarrow \infty$.

\subsection{Alternative Form of the Non-Parametric Linear MMSE}

Due to the fact that the exist of $R_{\mathrm{yy}}^{-1}$ is not always guaranteed, we provide the alternative form of the non-parametric linear MMSE filter. One can find that

$$
\begin{aligned}
\mathbf{v}_{d}^{H} & =\mathbf{h}_{d}^{H}\left(\Sigma^{-1}-\Sigma^{-1} \mathbf{h}_{d}\left(1+\Sigma_{h}\right)^{-1} \mathbf{h}_{d}^{H} \Sigma^{-1}\right) \\
& =\left(\mathbf{I}-\Sigma_{h}\left(1+\Sigma_{h}\right)^{-1}\right) \mathbf{h}_{d}^{H} \Sigma^{-1} \\
& =\left(1+\Sigma_{h}\right)^{-1} \mathbf{h}_{d}^{H} \Sigma^{-1}
\end{aligned}
$$

Using the Eigen-decomposition [11]

$$
\Sigma=\left[\begin{array}{ll}
\mathbf{U}_{S} & \mathbf{U}_{N}
\end{array}\right]\left[\begin{array}{cc}
\Lambda_{S}+\frac{1}{S N R} \mathbf{I} & 0 \\
0 & \frac{1}{S N R} \mathbf{I}
\end{array}\right]\left[\begin{array}{c}
\mathbf{U}_{S}^{H} \\
\mathbf{U}_{N}^{H}
\end{array}\right],
$$

then

$$
\Sigma^{-1}=\mathbf{U}_{S}\left(\Lambda_{S}+\frac{1}{S N R} \mathbf{I}\right)^{-1} \mathbf{U}_{S}^{H}+\operatorname{SNR}\left(\mathbf{U}_{N} \mathbf{U}_{N}^{H}\right)
$$


Plugging (15) into (13), we have

$$
\begin{aligned}
\mathbf{v}_{d}^{H} & =\frac{1}{S N R}\left(\frac{1}{S N R}+\frac{1}{S N R} \Sigma_{h}\right)^{-1} \mathbf{h}_{d}^{H} \Sigma^{-1} \\
& =\left(\frac{1}{S N R}+\frac{1}{S N R} \mathbf{h}_{d}^{H} \mathbf{F} \mathbf{h}_{d}+\mathbf{h}_{d}^{H} \mathbf{P}_{I}^{\perp} \mathbf{h}_{d}\right)^{-1} \mathbf{h}_{d}^{H}\left(\frac{1}{S N R} F+\mathbf{P}_{I}^{\perp}\right)
\end{aligned}
$$

where

$\mathbf{F}=\mathbf{U}_{S}\left(\Lambda_{S}+\frac{1}{S N R} \mathbf{I}\right)^{-1} \mathbf{U}_{S}^{H}$ and $\mathbf{P}_{I}^{\perp}=\mathbf{U}_{N} \mathbf{U}_{N}^{H}$. By employing the singular value decomposition $\mathbf{h}_{d}^{H} \mathbf{P}_{I}^{\perp}=\mathbf{V} \Lambda \mathbf{U}^{H}$ and choosing $\mathbf{W}$ such that the matrix $\left[\begin{array}{ll}\mathbf{V} & \mathbf{W}\end{array}\right]$ is unitary. Then, (16) can be resolved onto the basis $\left[\begin{array}{ll}\mathbf{V} & \mathbf{W}\end{array}\right]$ as follows

$$
\begin{aligned}
\mathbf{v}_{d}^{H} & =\left[\begin{array}{ll}
\mathbf{V} & \mathbf{W}
\end{array}\right]\left[\begin{array}{cc}
\frac{1}{S N R}\left(\mathbf{I}+\mathbf{V}^{H} \mathbf{h}_{d}^{H} \mathbf{F} \mathbf{h}_{d} \mathbf{V}\right)+\Lambda^{2} & 0 \\
0 & \frac{1}{S N R} \mathbf{I}
\end{array}\right]^{-1}\left[\begin{array}{c}
\mathbf{V}^{H} \\
\mathbf{W}^{H}
\end{array}\right] \mathbf{h}_{d}^{H}\left(\frac{1}{S N R} \mathbf{F}+\mathbf{P}_{I}^{\perp}\right) \\
& =\mathbf{V}\left(\frac{1}{S N R}\left(\mathbf{I}+\mathbf{V}^{H} \mathbf{h}_{d}^{H} \mathbf{F} \mathbf{h}_{d} \mathbf{V}\right)+\Lambda^{2}\right)^{-1} \mathbf{V}^{H} \mathbf{h}_{d}^{H}\left(\frac{1}{S N R} \mathbf{F}+\mathbf{P}_{I}^{\perp}\right)
\end{aligned}
$$

since $\mathbf{W}^{H} \mathbf{h}_{d}^{H}=0$ [11]. If we consider the interference-limited regime $\left(\frac{1}{\operatorname{SNR}} \rightarrow 0\right)$, we have

$$
\mathbf{v}_{d}^{H}=\mathbf{V} \Lambda^{-2} \mathbf{V}^{H} \mathbf{h}_{d}^{H} \mathbf{P}_{I}^{\perp}=\mathbf{V} \Lambda^{-1} \mathbf{U}^{H}=\left(\mathbf{P}_{I}^{\perp} \mathbf{h}_{d}\right)^{\dagger}=\left(\mathbf{h}_{d}^{H} \mathbf{P}_{I}^{\perp} \mathbf{h}_{d}\right)^{-1} \mathbf{h}_{d}^{H} \mathbf{P}_{I}^{\perp} .
$$

By letting $\quad R_{\mathbf{y y}}=R_{A}+\frac{1}{S N R} \mathbf{I}=A A^{H}+\frac{1}{S N R} \mathbf{I} \quad$ where $\quad R_{A}=\mathbf{h}_{d} \mathbf{h}_{d}^{H}+\sum \mathbf{h}_{i} \mathbf{h}_{i}^{H} \quad$ and $A=\left[\begin{array}{llll}\mathbf{h}_{d} & \mathbf{h}_{1} & \Lambda & \mathbf{h}_{n}\end{array}\right]$ [11]. Therefore, (18) can be expressed as

$$
\mathbf{v}_{d}^{H}=\left(\mathbf{h}_{d}^{H} R_{A}^{\dagger} \mathbf{h}_{d}\right)^{-1} \mathbf{h}_{d}^{H} R_{A}^{\dagger} .
$$

Since $R_{\mathrm{yy}}^{\dagger}=R_{A}^{\dagger}$ in the interference-limited regime, (9) can be reduced to

$$
\mathbf{v}_{d}=\hat{R}_{\mathbf{y y}}^{\dagger} \mathbf{h}_{d}\left(\mathbf{h}_{d}^{H} \hat{R}_{\mathbf{y y}}^{\dagger} \mathbf{h}_{d}\right)^{-1}
$$

where $\hat{R}_{\mathrm{yy}}=\frac{1}{M} \mathbf{Y} \mathbf{Y}^{H}$ is the sample correlation matrix obtained from the received signal set $\mathbf{Y}=\left[\begin{array}{llll}\mathbf{y}_{1} & \mathbf{y}_{2} & \Lambda & \mathbf{y}_{M}\end{array}\right]$.

Note that the desired transmitter should be turned off for $K$ symbols period in order to attain fairly good sample covariance matrix. Also note that no such requirement is necessary for the proposed approach in (21). While $K$ is the sampling overhead in the packet transmission, $M$ in (21) can be freely selected within the range of the packet length. 


\section{SIMULATION AND DISCUSSION}

In this section, we compare the transmission capacity of the proposed technique with the conventional MMSE, MMSE with imperfect CSI (MMSE with $K$ samples), as well as maximum ratio combining (MRC) and zero forcing (ZF) schemes (full ZF and partial ZF). While the MRC maximizes the desired signal power and the full $\mathrm{ZF}$ selects the filter orthogonal to $N-1$ interferer channels, the partial $\mathrm{ZF}$ employs some of the receive degrees of freedom for interference annihilation while exploiting the rest of degrees of freedom to boost desired signal power (readers are referred to [3] for details).

The simulation setup is based on the 2-D PPP transmitters which are realized on the square distances. The SINR and outage probability are computed and compared to determine maximum density over several thousand iterations. We assume that the elements of each transmitter's channel vectors are i.i.d. zero mean complex Gaussian random variables with unit variance, which almost surely ensures that the desired channel $\mathbf{h}_{d}$ and interferer channels $\left\{\mathbf{h}_{i}\right\}_{i=1}^{\mathrm{A}(\lambda)}$ have full column rank. For comparison, the number of cancelled interferers $\theta N$ is considered in the partial ZF [3].

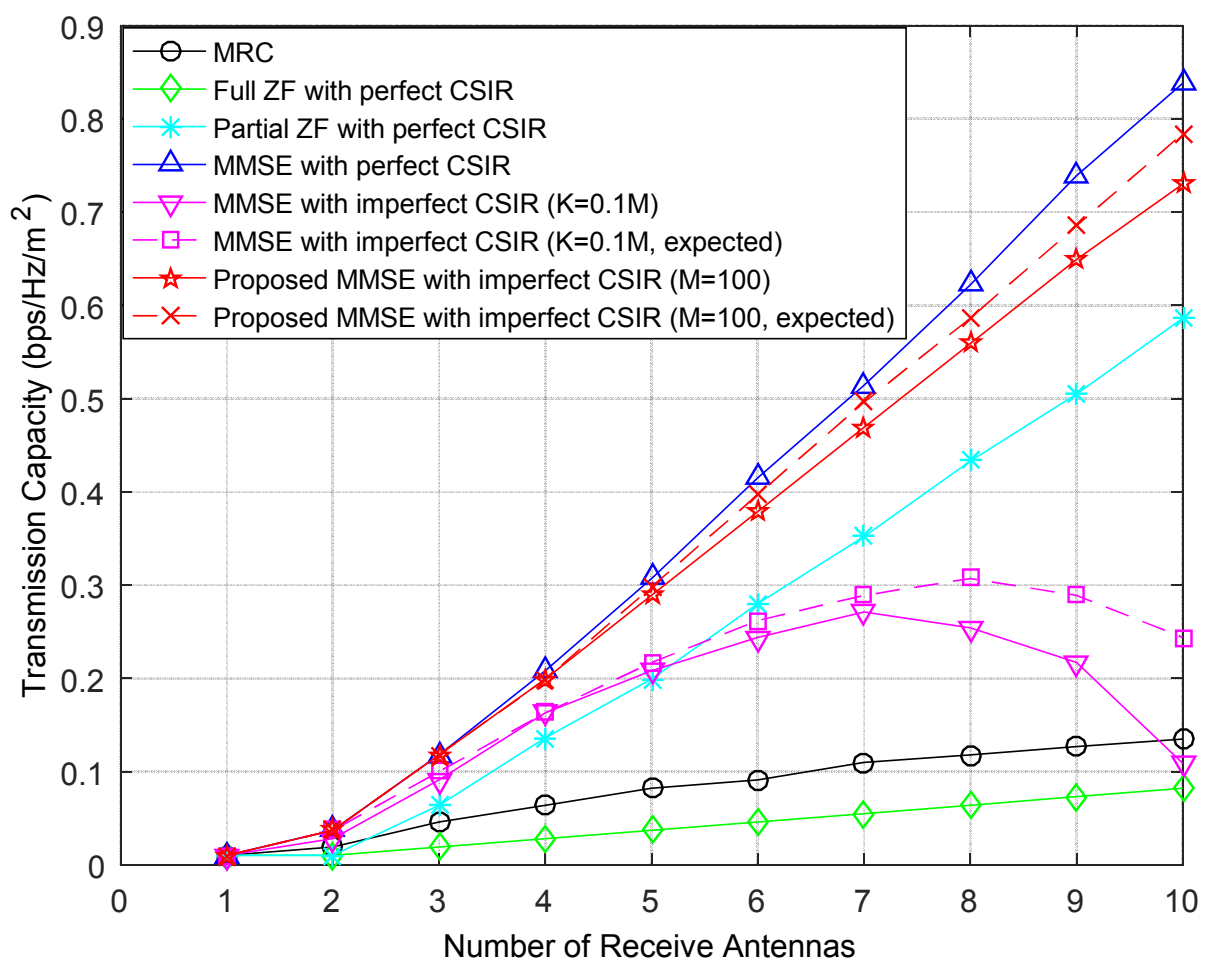

Figure 3. Transmission Capacity versus $N$ for $\varepsilon=0.1, \beta=1, \alpha=3, d=1$, and $K=10$.

In Fig. 3, we plot the transmission capacity as a function of $N$. Note that $K=10$ (10\% of packet length) and $M$ is the packet length. We observe that the proposed scheme, MMSE, and PZF show linearly increasing transmission capacity, whereas MMSE with $K$ samples, MRC, and full ZF exhibit much poorer scaling. In particular, although the proposed filter leaves a performance gap from MMSE, the transmission capacity of the proposed method is larger than the MMSE with $K$ samples and PZF, and the gain gets larger as $N$ increases. Due to the scaling factor of 
$\left(1-\frac{N-1}{K+1}\right)$, the expected SINR of the MMSE with $K$ samples is smaller than that of the MMSE with full CSI, the transmission capacity of the MMSE with $K$ samples is decreased when $K$ is a fixed number and $N$ goes to large number.

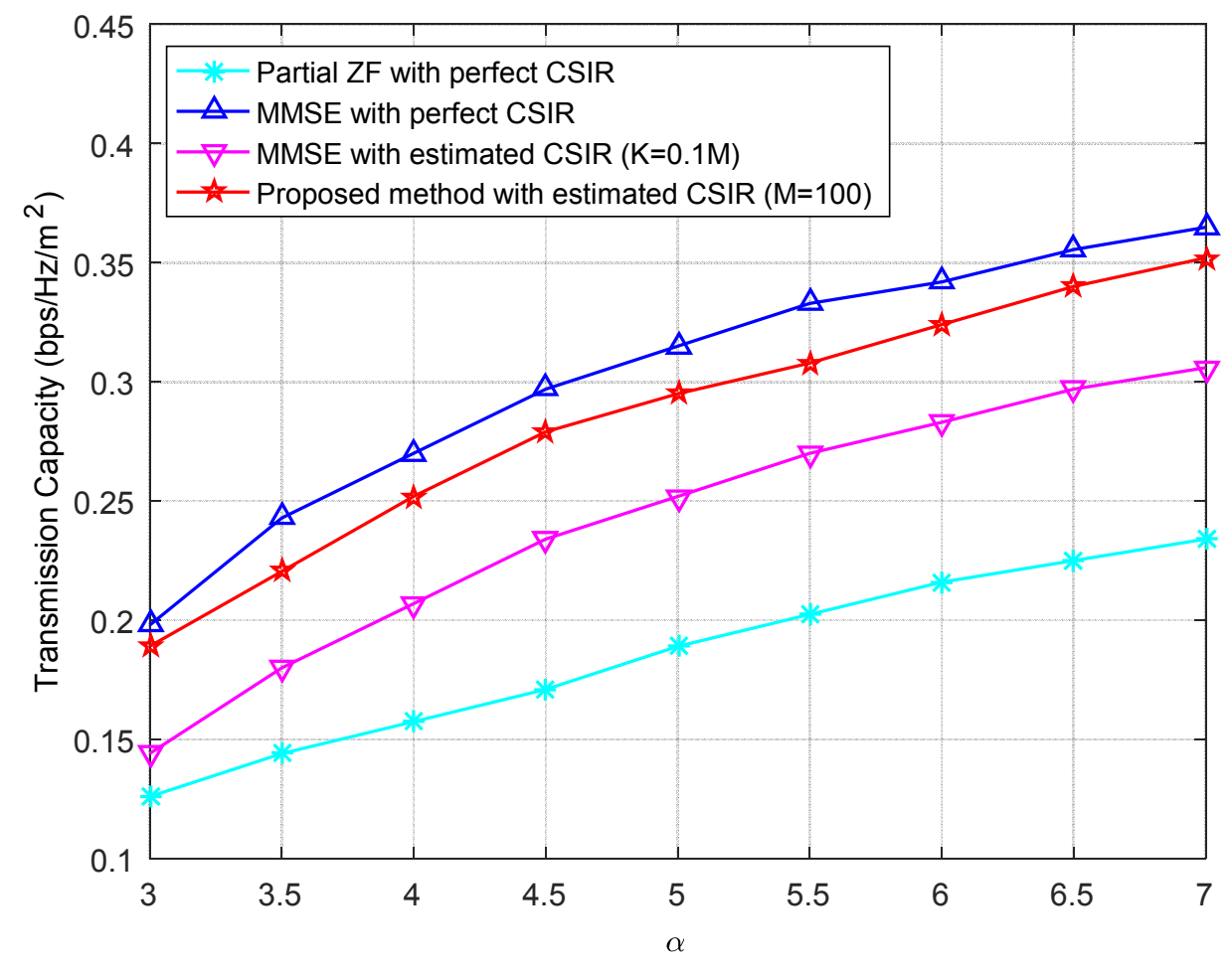

Figure 4. Transmission Capacity versus $\alpha$ for $\mathcal{E}=0.1, \beta=1, d=1, N=6$, and $S N R=10 d B$.

In Fig. 4, we plot the transmission capacity as a function of path loss exponent. The transmission capacity increases with the path loss exponent due to the fact that while the quality of the desired Tx-Rx pair is reduced by a higher $\alpha$, the effect of interference is also decreased. Note that the proposed method outperforms the MMSE with $K$ samples and partial ZF at all path loss exponent regime. This result shows that this interference degradation has a more significant effect on the ad hoc network system.

Finally, in order to solidify our conclusions, we plot the transmission capacity as a function of the number of blocks. From Fig. 5, we observe that the transmission curves of the MMSE and partial $\mathrm{ZF}$ are consistent due to full CSI structure. On the contrary, the transmission capacities of the proposed method and MMSE with $K$ samples are increasing function of the number of blocks since the accuracy of the covariance matrix is increased when the number of blocks grows. Although the transmission capacity of the MMSE with $K$ samples is close to the that of the optimal MMSE filter, it is impractical since the transmission data rate loss also increases. The results indicate that the proposed method is competitive option in real ad hoc network scenarios. 


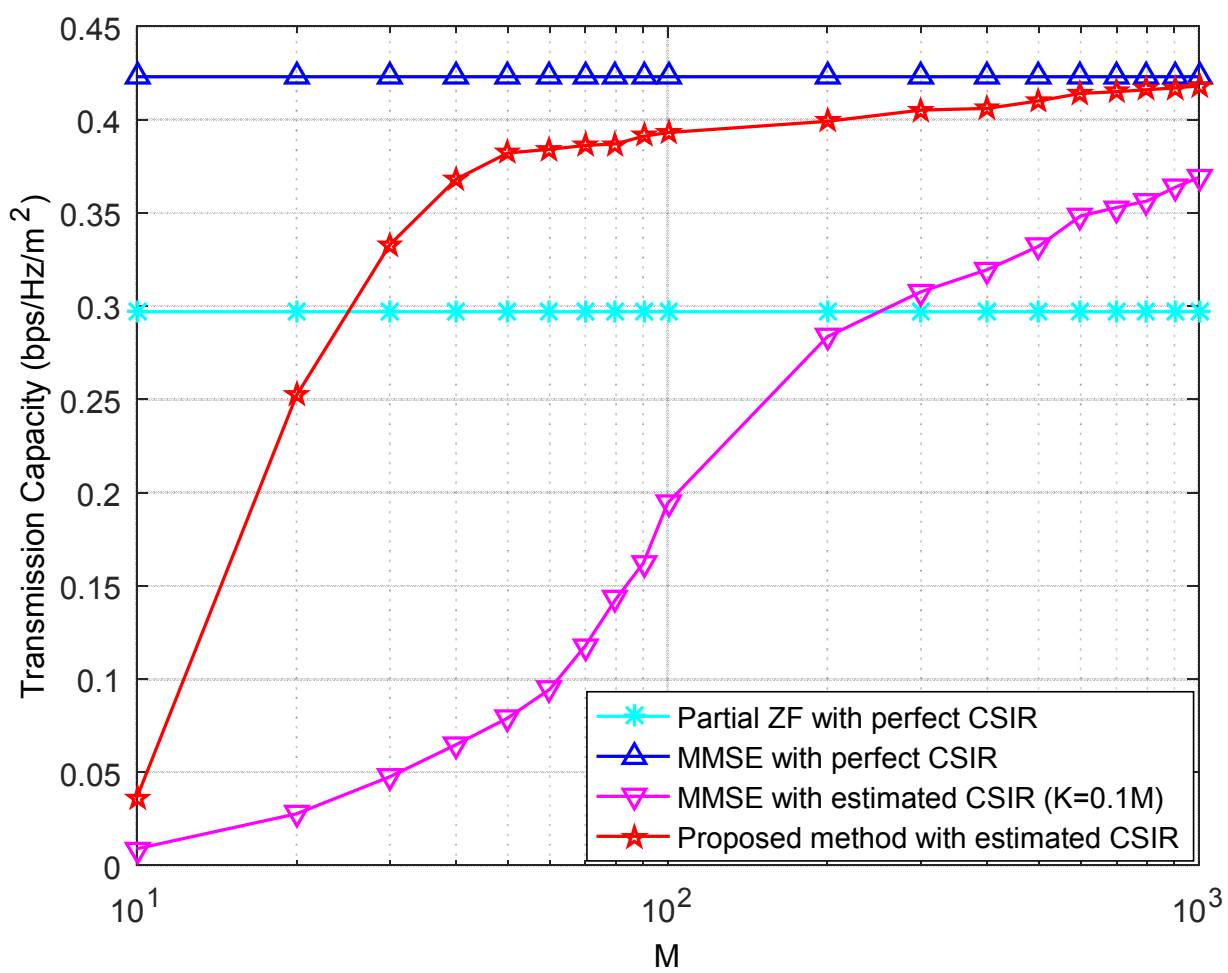

Figure 5. Transmission Capacity versus number of samples for $\varepsilon=0.1, \beta=1, \alpha=3, d=1, N=6$, and $S N R=10 \mathrm{~dB}$.

\section{CONCLUSION}

In this paper, we investigated an approach based on MMSE filter achieving robustness of real ad hoc network in which the CSIR of all interferers is unavailable. Motivated by the fact that the MMSE with imperfect CSIR brings significant transmission rate loss due to the inactive mode of the desired transmitter, we employed the non-parametric linear MMSE filter to achieve large fraction of the MMSE filter transmission capacity. We observe from the transmission capacity performance that the proposed method outperforms the MMSE receiver under imperfect CSIR condition and conventional receive antenna algorithms. Future study needs to be directed towards the investigation of the performance when the interfering transmitters are heterogeneous.

\section{ACKNOWLEDGEMENTS}

This work was supported by the National Research Foundation of Korea (NRF) grant funded by the Korean government(MSIP)(2014R1A5A1011478) and the ICT R\&D program of MSIP/IITP[B0717-16-0023, Fundamental technology development of transmission, modulation, and coding techniques for low-power and low-complexity 5G-based IoT environments with massive connectivity]. 


\section{REFERENCES}

[1] A. Hunter, J. G. Andrews, and S. Weber, "The transmission capacity of ad hoc networks with spatial diversity,” IEEE Trans. Wireless Commun., vol. 7, no. 12, pp. 5058-5071, Dec. 2008.

[2] K. Huang, J. G. Andrews, D. Guo, R. W. Heath, Jr., and R. Berry, "Spatial interference cancellation for multi-antenna mobile ad hoc networks," IEEE Trans. Inf. Theory, submitted. [Online]. Available:arxic.org/abs/0807.1773v2

[3] N. Jindal, J. G. Andrews, and S. weber, "Multi-antenna communication in ad hoc networks: achieving MIMO gains with SIMO transmission,” IEEE Trans. Comm., vol. 59, no. 2, pp. 529-540, Feb. 2011.

[4] S. Weber, J. G. Andrews, and N. Jindal, "An overview of the transmission capacity of wireless networks,” IEEE Trans. Comm., vol. 58, no. 12, pp. 3593-3604, Dec. 2010.

[5] M. Haenggi, "On distances in uniformly random networks," IEEE Trans. Inf. Theory, vol. 51, no. 10, pp. 3584-3586, Oct. 2005.

[6] S. Weber, X. Yang, J. G. Andrews, and G. de Veciana, "Transmission capacity of wireless ad hoc networks with outage constraints," IEEE Trans. Inf. Theory, vol. 51, no. 12, pp. 4091-4102, Dec. 2005.

[7] J. Blomer and N. Jindal, "Transmission capacity of wireless ad hoc networks: successive interference cancellation vs. joint detection," Proc. IEEE Intl. Conf. Commun. (ICC), Dresden, Germany, June 2009.

[8] S. Govindasamy, D. W. Bliss, and D. H. Staelin, "Spectral efficiency in single-hop ad-hoc network wireless netowrks with interference using adaptive antenna arrays," IEEE J. Sel. Areas Commun., vol. 25, no. 7, pp. 1358-1369, Sep. 2007.

[9] I. Reed, J. Mallet, and L. Brennan, "Rapid convergence rate in adaptive arrays," IEEE Trans. Aerospace Electron. Syst., vol. 10, no. 6, pp. 853-863, Nov. 1974.

[10] S.\M.\Kay, Fundamentals of Statistical Signal Processing: Estimation Theory, Prentice Hall, 1998.

[11] L. Scharf and M. McCloud, "Blind adaptation of zero forcing projections and oblique pseudo-inverses for subspace detection and estimation when interference dominates noise," IEEE Trans. Sig. Proc., vol. 50, no. 12, pp. 2938-2946, Dec. 2002.

\section{AUTHORS}

Sunho Park received the B.S., M.S., and Ph.D. degrees from the School of Information and Communication from Korea University, Seoul, in 2008, 2010, and 2015, respectively. From September 2015 to September 2016, he was with Institute of New Media and Communications, Seoul National University, as a Senior Researcher. $\mathrm{He}$ is currently a research assistant professor in Seoul National University, Seoul, Korea. His research interests include wireless communications and signal processing.

Byonghyo Shim received the B.S. and M.S. degrees in control and instrumentation engineering from Seoul National University, Korea, in 1995 and 1997, respectively. He received the M.S. degree in mathematics and the Ph.D. degree in electrical and computer engineering from the University of Illinois at Urbana-Champaign (UIUC), USA, in 2004 and 2005, respectively.

From 1997 and 2000, he was with the epartment of Electronics Engineering, Korean

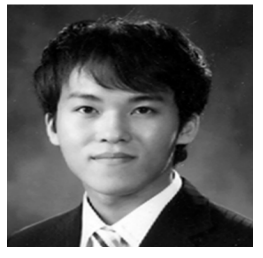
Air Force Academy as an Officer (First Lieutenant) and an Academic Full-time Instructor. From 2005 to 
2007, he was with Qualcomm Inc., San Diego, CA, USA, as a Staff Engineer. From 2007 to 2014, he was with the School of Information and Communication, Korea University, Seoul, as an Associate Professor. Since September 2014, he has been with the Department of Electrical and Computer Engineering, Seoul National University, where he is presently an Associate Professor. His research interests include wireless communications, statistical signal processing, estimation and detection, compressive sensing, and information theory.

Dr. Shim was the recipient of the 2005 M. E. Van Valkenburg Research Award from the Electrical and Computer Engineering Department of the University of Illinois and 2010 Hadong Young Engineer Award from IEIE. He is currently an Associate Editor of the IEEE WIRELESS COMMUNICATIONS LETTERS, Journal of Communications and Networks, and a Guest Editor of the IEEE JOURNAL ON SELECTED AREAS IN COMMUNICATIONS (JSAC). 